\title{
Retenção e disponibilidade de água às plantas, em solo sob plantio direto e preparo convencional
}

Genei A. Dalmago ${ }^{1}$, Homero Bergamaschi2 ${ }^{2}$, João I. Bergonci ${ }^{3}$, Cleusa A. M. B. Krüger ${ }^{4}$, Flávia Comiran ${ }^{2} \&$ Bruna M. M. Heckler ${ }^{2}$

RESUMO

Teve-se, neste trabalho, como função principal, avaliar a retenção de água no solo e sua disponibilidade às plantas, em plantio direto (PD) e preparo convencional (PC). 0 estudo foi conduzido em Eldorado do Sul, Brasil, em 2002. Densidade do solo, porosidade total, macroporosidade, mesoporosidade, microporosidade, distribuição do tamanho de mesoporos, curvas de retenção de água, capacidade de campo (CC) e ponto de murcha permanente (PMP) foram analisados em sete profundidades no perfil. As maiores diferenças ocorreram próximo à superfície. Na profundidade de $2,5 \mathrm{~cm}$ a densidade foi $7 \%$ menor e a porosidade total foi cerca de 15\% maior em PD do que em PC. A mesoporosidade apresentou distribuição exponencial em PD, com valores mais elevados em tamanhos maiores de poros, mas teve tendência a uma curva normal em PC. Em todo o perfil, PC apresentou cerca de 53\% mais água armazenada em comparação a PD; entretanto, a armazenagem de água foi $80 \%$ maior em PD próximo à superfície (2,5 $\mathrm{cm}$ de profundidade). Nos primeiros $15 \mathrm{~cm}$ de profundidade $70 \%$ da água disponível foram retidos acima de - 80 kPa em PD, em comparação a 50\%, para PC. 0 sistema plantio direto aumenta a disponibilidade de água às plantas próximo à superfície e com menos energia de retenção, em comparação com o preparo convencional.

Palavras-chave: água disponível, manejo do solo, armazenagem de água no solo, propriedades físicas, semeadura direta

\section{Retention and availability of water to plants in soils under no-tillage and conventional tillage systems}

\begin{abstract}
This work had the objective of evaluating the soil water storage and availability to plants, when comparing the no-tillage (NT) and conventional tillage (CT) systems. The study was conducted in Eldorado do Sul, Brazil, in 2002. Soil density, total porosity, macroporosity, mesoporosity, microporosity, distribution of mesopores size, curves of water retention, field capacity $(F C)$, and permanent wilting point (PWP) were analyzed in seven depths, into the soil profile. The highest differences occurred close to the soil surface. At $2.5 \mathrm{~cm}$ deep, the soil density was $7 \%$ lower while the total soil porosity was about $15 \%$ higher in NT than in CT. The mesoporosity showed an exponential distribution in NT, with highest values for largest mesopores, but it tended to a normal curve in CT. Considering the entire soil profile, the soil water storage was about $53 \%$ higher in CT than in NT. However, close to the soil surface (at $2.5 \mathrm{~cm}$ deep) it was $80 \%$ higher in NT than in CT. From the soil surface to $15 \mathrm{~cm}$ deep, $70 \%$ of the available water was retained above the limit of $-80 \mathrm{kPa}$ in NT, in comparison to $50 \%$ in $\mathrm{CT}$. The no-tillage system increases the water availability to plants and reduces the energy of retention in the upper soil layers, in comparison to the conventional tillage.
\end{abstract}

Key words: available water, soil management, soil water storage, physical properties, no-tillage

${ }^{1}$ Embrapa Trigo, BR 285, km 294, CP 451, CEP 99001-970 Passo Fundo, RS. Fone (54) 3316-5836, Fax (54) 3316-5802. E-mail: dalmago@cnpt.embrapa.br ${ }^{2}$ Faculdade de Agronomia/UFRGS, Av. Bento Gonçalves 7712, CP 15100, CEP 91501-970, Porto Alegre, RS. Fone (51) 3308-6255. E-mail: homerobe@ufrgs.br; flaviacomiran@pop.com.br; bruheckler@hotmail.com

${ }^{3}$ D epartamento de Botânica/U FRGS, Av. Paulo Gama s.n. CEP 90046-900, Porto Alegre, RS. Fone (51) 3308-7653. E-mail: joao.bergonci@ufrgs.br ${ }^{4}$ D epartamento de Estudos Agrários/U NIJUil, Rua do Comércio 3000, CEP 98700-000, ljuí, RS. Fone (55) 3332-0420. E-mail: cleusa.bianchi@unijui.edu.br 


\section{INTRODUÇãO}

A mudança na forma de manejo do solo, e de preparo convencional (PC) para plantio direto (PD), altera propriedades físicas e hídricas do solo, haja vista que, normalmente, solos cultivados em PD sofrem compactação na superfície devido ao tráfego de máquinas e implementos agrícolas e consolidação natural pelo não revolvimento, enquanto solos cultivados em PC são frequentemente revolvidos, o que modifica suas propriedades físico-hídricas em curto espaço de tempo (Tormena et al., 1998; 2004).

Em geral, as diferenças de cultivo fazem com que a densidade do solo seja mais elevada, sobretudo nos primeiros anos de implantação do sistema PD em relação ao PC. Aumento mais significativo é comum junto à superfície, até cerca de $20 \mathrm{~cm}$ de profundidade (Stone \& Silveira, 2001; Cruz et al., 2003; Tormena et al., 2004; Osunbitan et al., 2005; Secco et al., 2005; Bescansa et al., 2006; Moret \& Arrúe, 2007), dependendo de como o sistema foi manejado, do tipo de solo, do histórico da área e de como o PD foi iniciado (Stone \& Silveira, 2001). Como consequência do aumento da densidade ocorre redução da porosidade total, na maioria das vezes pela diminuição da macroporosidade, o que aumenta a microporosidade quando relacionados com solo em PC (Stone \& Silveira, 2001; Cruz et al., 2003; Tormena et al., 2004; Secco et al., 2005). Ademais, o não revolvimento do solo em PD e a existência de bioporos tornam a porosidade mais eficiente no transporte líquido e gasoso em relação ao convencional, devido à manutenção da continuidade dos poros, a qual é quebrada no solo em PC (Osunbitanet al., 2005; Hubert et al., 2007).

As diferenças de densidade e na dinâmica porosa entre o solo sob PC e PD, afetam a retenção de água no mesmo (Bescansa et al., 2006), principalmente pelo efeito sobre o fenômeno da capilaridade. A capilaridade está ligada à afinidade das partículas do solo com a água e depende da geometria porosa do meio (forma, tamanho, orientação e distribuição dos poros), que é afetada pelo sistema de manejo adotado (Rasiah \& Aylmore, 1998). A adsorção, outro fenômeno ligado à retenção de água, está fortemente relacionada à textura do solo (Hillel, 1998), a qual não é afetada significativamente por diferentes sistemas de manejo em um mesmo solo.

A relação entre a energia com que a água está retida nos constituintes do solo e o seu conteúdo, é expressa pela curva característica de retenção de água. Com base no seu ajuste, Rojas \& Lier (1999) observaram diferenças significativas de retenção entre o mesmo solo em PD e PC, em praticamente todos os potenciais avaliados e em distintas camadas de solo, com valores sistematicamente maiores em PC, condição também observada por Shukla et al. (2003), porém em outro tipo de solo e local, Bescansa et al. (2006) verificaram retenção de água mais elevada em $\mathrm{PD}$, em potenciais menores que $0 \mathrm{kPa}$. Por outro lado, Tormena \& Silva (2002) e Xu \& Mermonud (2003) não notaram influência significativa do sistema de manejo do solo no ajuste de curvas características, indicando a mesma capacidade de retenção de água. A elevação na retenção de água em PD se dá, em geral, pelo aumento da mesoporosidade (Rojas \& Lier, 1999) e do teor de matéria orgânica, a qual tem maior capacidade de hidratação (Model et al., 1995) e tem efeito positivo na conservação das propriedades físico-hídricas do solo (Masri \& Ryan, 2006); entretanto, o aumento na retenção de água pelo solo não implica, necessariamente, em maior disponibilidade às plantas (Tollner et al., 1984), uma vez que a disponibilidade está relacionada aos limites de capacidade de campo (CC) e do ponto de murcha permanente (PMP), modificados pelo manejo do solo.

Apesar de existirem resultados divergentes na literatura, a maioria dos trabalhos aponta para maior armazenagem e disponibilidade de água às plantas em solo sob PD em relação a PC (Salton \& Mielniczuk, 1995; Drury et al., 1999; Lampurlanés et al., 2001, Bescansa et al., 2006; Vita et al., 2007; Zanette et al., 2007), o que tem sido constatado principalmente em sistemas que apresentam mais tempo de utilização e em camadas próximas à superfície, possivelmente pelo efeito de longo prazo do PD sobre a densidade e sobre a porosidade do solo (Kay \& Vandenbygaart, 2002). Maior retenção e disponibilidade de água às plantas no solo em PD, normalmente são atribuídas à barreira formada pelos resíduos (palha), que reduziria a evaporação da água na superfície (Hatfield et al., 2001; Baumhardt \& Jones, 2002) e à modificação da geometria porosa do solo (Tollner et al., 1984). As diferenças na morfologia porosa do solo entre o sistema de plantio direto e o preparo convencional, foram descritas por Hubert et al. (2007).

Conhecer a forma e a magnitude das alterações nas propriedades físico-hídricas do solo permite melhorar a compreensão das relações existentes neste sistema de manejo e a avaliação de seus efeitos sobre processos associados; também, pode possibilitar ajustes de índices e modelos gerados, por exemplo, em PC, permitindo o uso adequado dos mesmos em outros sistemas de manejo; neste sentido, o objetivo neste trabalho foi avaliar alterações na retenção de água no solo e na sua disponibilidade às plantas, ao longo do perfil, em sistema plantio direto, em comparação com o preparo convencional.

\section{MATERIAL E MÉTODOS}

O experimento foi realizado na Estação Experimental Agronômica da Universidade Federal do Rio Grande do Sul, em Eldorado do Sul, RS ( $30^{\circ} 05^{\prime} \mathrm{S}, 51^{\circ} 39^{`} \mathrm{~W}, 40 \mathrm{~m}$ altitude). O clima da região é subtropical úmido de verão quente do tipo Cfa (Köeppen) (Bergamaschi et al., 2003). O solo da área é um Argissolo Vermelho Distrófico típico, com horizonte B textural (Rojas, 1998).

A área experimental é plana e foi planejada, originalmente, na forma de experimento em faixas, conforme os pressupostos de Hanks et al. (1980) para testar a aplicação de diferentes lâminas de irrigação na parte central da área experimental. Transversalmente às faixas foram dispostos, em 1995, os sistemas de manejo de solo em plantio direto (PD) e preparo convencional (PC), em blocos ao acaso com cinco repetições. Para efeito deste trabalho se utilizaram apenas três blocos (repetições), cujos fatores foram sistema de manejo de solo e profundidade no perfil e os tratamentos foram os sistemas PD e PC e as profundidades de 2,5, 7,5, 15, 30, 45, 60 e $75 \mathrm{~cm}$. 
As parcelas, tanto em PD quanto em PC, sempre foram cultivadas com milho no verão e mistura de aveia preta (Avena strigosa) e ervilhaca (Vicia sativa) no inverno. A cobertura de inverno foi semeada sem revolvimento do solo, enquanto a semeadura do milho em PD se deu após a dessecação da cultura de inverno, que foi acamada ao solo; em PC, a semeadura do milho foi feita após a incorporação da cobertura de inverno por uma aração na profundidade de 18 a $25 \mathrm{~cm}$ e duas gradagens. Em referência à coleta de solo, abriram-se trincheiras até um metro de profundidade no final do ciclo da cultura de inverno, em 2002. Em todas as profundidades se coletaram amostras para determinação de propriedades físico-hídricas do solo: densidade, porosidade (total, macro, meso e microporosidade) e curvas de retenção de água. A granulometria foi determinada por Rojas (1998), no terceiro ano de implantação dos sistemas de manejo, tendo apresentado 49, 22, $29 \%$ em PD e 42, 27, 31\% em PC de areia, silte e argila, respectivamente (médias de até $110 \mathrm{~cm}$ de profundidade).

As amostras de solo para determinação das curvas características de retenção de água foram coletadas com cilindros de 2,5 cm de altura e $6,4 \mathrm{~cm}$ de diâmetro. Utilizaram-se um funil tipo "Haines" para determinação da umidade no potencial matricial de -6 kPa e uma câmara de pressão com placa porosa (Câmara de Richards) para potenciais de -33, -100, -300, 500 e $-1500 \mathrm{kPa}$. Os dados de umidade volumétrica $(\theta v)$ e respectivos potenciais foram ajustados à equação de van Genuchten:

$$
\theta \mathrm{v}=\theta \mathrm{r}+\frac{\theta \mathrm{s}-\theta \mathrm{r}}{\left[1+(\alpha \mid \psi \mathrm{ml})^{\mathrm{n}}\right]^{\mathrm{m}}}
$$

sendo $\theta \mathrm{v}\left(\mathrm{cm}^{3} \mathrm{~cm}^{-3}\right)$ a umidade volumétrica, $\Psi \mathrm{m}(\mathrm{kPa})$ o potencial matricial da água no solo, $\theta \mathrm{r}\left(\mathrm{cm}^{3} \mathrm{~cm}^{-3}\right)$ a umidade residual e $\theta \mathrm{s}\left(\mathrm{cm}^{3} \mathrm{~cm}^{-3}\right)$ a umidade de saturação, enquanto $\alpha$ $\left(\mathrm{kPa}^{-1}\right), \mathrm{m}$ e $\mathrm{n}$ são parâmetros empíricos.

Para a determinação da densidade do solo, coletaram-se amostras com cilindros de $7 \mathrm{~cm}$ de altura e $8,5 \mathrm{~cm}$ de diâmetro, exceto nas profundidades de 2,5 e $7,5 \mathrm{~cm}$, em que se utilizaram cilindros de $2,5 \mathrm{~cm}$ de altura e $6,4 \mathrm{~cm}$ de diâmetro. A densidade foi calculada pela razão entre a massa de solo seco e o volume da amostra, seguindo-se metodologia descrita por Blake et al. (1986).

A porosidade total foi calculada pela relação:

$$
\mathrm{Pt}=\left(1-\frac{\mathrm{Ds}}{\mathrm{Dp}}\right)
$$

sendo $\mathrm{Pt}\left(\mathrm{cm}^{3} \mathrm{~cm}^{-3}\right)$ a porosidade total, Ds $\left(\mathrm{g} \mathrm{cm}^{-3}\right)$ a densidade do solo e Dp $\left(\mathrm{g} \mathrm{cm}^{-3}\right)$ a densidade de partícula estimada para cada profundidade, de acordo com medições feitas por Rojas (1998); já a macroporosidade foi obtida pela diferença entre Pt e o volume de água $\left(\mathrm{cm}^{3} \mathrm{~cm}^{-3}\right)$ extraído no potencial matricial de $-6 \mathrm{kPa}$ (poros maiores que $50 \mu \mathrm{m}$ ), das amostras de determinação das curvas características de retenção de água. A umidade retida a partir do potencial de $-1500 \mathrm{kPa}$ foi considerada correspondente à microporosidade (poros menores que $0,2 \mu \mathrm{m}$ ) e a diferença entre a macro e a microporosi- dade foi acatada como mesoporosidade (poros entre 50 e 0,2 $\mu \mathrm{m}$ ), a qual foi dividida em cinco classes de tamanho de poros cujos limites foram: 50 a $8,9 \mu \mathrm{m}, 8,9$ a $2,9 \mu \mathrm{m}, 2,9$ a 1,0 $\mu \mathrm{m}, 1,0$ a $0,6 \mu \mathrm{m}$ e 0,6 a 0,2 $\mu \mathrm{m}$. Calculou-se o diâmetro de mesoporos pelo modelo de ascensão capilar de água (Libardi, 1995), considerando-se os potenciais matriciais de $-33,-100,-300,-500$ e $-1500 \mathrm{kPa}$, respectivamente, como limite inferior de cada classe.

A capacidade de campo (CC) foi determinada no campo, através da inundação de uma área de $9 \mathrm{~m}^{2}$, delimitada por chapas galvanizadas, até o solo atingir saturação completa; depois, a superfície foi coberta com lona plástica para evitar a evaporação e transcorridas as $72 \mathrm{~h}$ necessárias para a drenagem natural, três repetições de amostras de solo foram coletadas com trado holandês, em cada profundidade, respectivamente, de 2,5, 7,5, 15, 30, 45, 60 e $75 \mathrm{~cm}$. As amostras foram acondicionadas em latas de alumínio para secagem em estufa a $105^{\circ} \mathrm{C}$ até massa constante, a fim de se calcular a umidade gravimétrica, a qual foi transformada em $\theta \mathrm{v}$ através da multiplicação pela densidade do solo de cada profundidade. O ponto de murcha permanente (PMP) foi considerado a umidade residual no potencial matricial de $-1500 \mathrm{kPa}$.

Os resultados foram submetidos à análise de variância, aplicando-se o teste de Tukey com 5\% de probabilidade de erro, para comparação das médias.

\section{RESULTADOS E DISCUSSÃO}

A análise de variância mostrou interação significativa para uma parte das propriedades fisico-hídricas do solo (Tabela 1), enquanto para a outra parte (Tabela 2) a interação não foi significativa; portanto, houve efeito diferenciado de sistemas de manejo em profundidades apenas para um grupo de variáveis. Interação significativa entre diferentes sistemas de manejo de solo e profundidades de amostragem, também foi obtida por Tormena et al. (2004) para algumas propriedades físicas do solo.

A densidade do solo foi significativamente menor em PD mas só na primeira e na última profundidade, em relação ao PC, contrariando, em parte, a tendência de maior densidade do solo em PD próximo à superfície, relatada pela maior parte da bibliografia (Stone \& Silveira, 2001; Tormena et al., 2004; Bescansa et al., 2006; Moret \& Arrúe, 2007). O valor mais elevado de Ds em PD foi de $1,77 \mathrm{~g} \mathrm{~cm}^{-3}$ e foi estatisticamente diferente daquele observado no solo em PC, isto é, $1,66 \mathrm{~g} \mathrm{~cm}^{-}$ 3; ambos os valores foram observados na profundidade de $7,5 \mathrm{~cm}$; para a profundidade de $2,5 \mathrm{~cm}$ a diferença pode ser atribuída ao conteúdo mais elevado de matéria orgânica em $\mathrm{PD}$, a qual pode contribuir para redução da Ds em longo prazo (Tormena et al., 2002) por proporcionar maior e melhor agregação do solo (Razafimbelo et al., 2008); na profundidade de 7,5 cm a tendência de maior Ds em PD pode ser consequência da compactação do solo em $\mathrm{PD}$, causada pelo não revolvimento e tráfego de máquinas na superfície (Tormena et al., 2004).

Com relação à porosidade total do solo, ocorreu tendência inversa à densidade, conforme previsto (Tabela 1). Nas 
Tabela 1. Densidade (Ds), porosidade (total, macro, meso e micro), distribuição do tamanho de mesoporos, umidade no ponto de murcha permanente (PMP), em diferentes profundidades do solo sob plantio direto (PD) e preparo convencional (PC)

\begin{tabular}{|c|c|c|c|c|c|c|c|c|}
\hline \multirow{2}{*}{$\begin{array}{l}\text { Sistema de } \\
\text { Manejo do solo }\end{array}$} & \multirow[t]{2}{*}{ Ds $\left(\mathrm{g} \mathrm{cm}^{-3}\right)$} & \multicolumn{4}{|c|}{ Porosidade do solo $\left(\mathrm{cm}^{3} \mathrm{~cm}^{-3}\right)$} & \multicolumn{2}{|c|}{$\begin{array}{l}\text { Distribuição do tamanho de } \\
\text { mesoporos }\left(\mathrm{cm}^{3} \mathrm{~cm}^{-3}\right)\end{array}$} & \multirow[t]{2}{*}{ PMP $\left(\mathrm{cm}^{3} \mathrm{~cm}^{-3}\right)$} \\
\hline & & Total & Macro & Meso & Micro & Classe1 & Classe4 & \\
\hline \multicolumn{9}{|c|}{ Profundidade $-2,5 \mathrm{~cm}$} \\
\hline PD & $1,63 \mathrm{~b}$ & $0,373 a$ & $0,067 \mathrm{a}$ & 0,177 a & $0,128 \mathrm{a}$ & $0,099 a$ & $0,010 \mathrm{a}$ & $0,147 a$ \\
\hline PC & $1,75 a$ & $0,319 b$ & $0,071 \mathrm{a}$ & $0,086 \mathrm{~b}$ & $0,163 \mathrm{a}$ & $0,014 \mathrm{~b}$ & $0,011 \mathrm{a}$ & $0,193 \mathrm{a}$ \\
\hline \multicolumn{9}{|c|}{ Profundidade - 7,5 cm } \\
\hline PD & $1,77 \mathrm{a}$ & $0,321 \mathrm{a}$ & $0,085 a$ & $0,115 a$ & $0,121 \mathrm{a}$ & $0,048 \mathrm{a}$ & $0,009 a$ & $0,128 \mathrm{a}$ \\
\hline PC & $1,66 \mathrm{~b}$ & $0,441 \mathrm{a}$ & $0,106 \mathrm{a}$ & 0,069 a & $0,266 \mathrm{a}$ & $0,034 \mathrm{a}$ & $0,011 \mathrm{a}$ & $0,162 \mathrm{a}$ \\
\hline \multicolumn{9}{|c|}{ Profundidade $-15 \mathrm{~cm}$} \\
\hline PD & $1,74 \mathrm{a}$ & $0,327 a$ & $0,112 \mathrm{a}$ & $0,058 \mathrm{a}$ & $0,156 a$ & $0,017 \mathrm{a}$ & $0,009 a$ & $0,159 a$ \\
\hline $\mathrm{PC}$ & $1,73 \mathrm{a}$ & $0,421 \mathrm{a}$ & $0,120 \mathrm{a}$ & $0,141 \mathrm{a}$ & $0,160 \mathrm{a}$ & $0,016 \mathrm{a}$ & $0,014 \mathrm{a}$ & $0,171 \mathrm{a}$ \\
\hline \multicolumn{9}{|c|}{ Profundidade $-30 \mathrm{~cm}$} \\
\hline PD & $1,70 \mathrm{a}$ & $0,340 \mathrm{~b}$ & $0,109 a$ & $0,072 \mathrm{a}$ & $0,159 a$ & $0,023 \mathrm{a}$ & $0,016 \mathrm{a}$ & $0,174 a$ \\
\hline $\mathrm{PC}$ & $1,49 \mathrm{~b}$ & $0,421 \mathrm{a}$ & $0,120 \mathrm{a}$ & $0,141 \mathrm{a}$ & $0,160 \mathrm{a}$ & $0,041 \mathrm{a}$ & $0,007 \mathrm{~b}$ & $0,164 \mathrm{a}$ \\
\hline \multicolumn{9}{|c|}{ Profundidade $-45 \mathrm{~cm}$} \\
\hline PD & $1,68 \mathrm{a}$ & $0,358 \mathrm{a}$ & $0,099 a$ & $0,124 a$ & $0,134 \mathrm{a}$ & $0,106 a$ & $0,006 \mathrm{a}$ & $0,149 a$ \\
\hline $\mathrm{PC}$ & $1,62 \mathrm{a}$ & $0,372 \mathrm{a}$ & $0,042 b$ & 0,168 a & $0,162 \mathrm{a}$ & $0,106 \mathrm{a}$ & $0,002 \mathrm{a}$ & $0,162 \mathrm{a}$ \\
\hline \multicolumn{9}{|c|}{ Profundidade - $60 \mathrm{~cm}$} \\
\hline PD & $1,58 \mathrm{a}$ & $0,393 \mathrm{a}$ & $0,131 \mathrm{a}$ & $0,081 b$ & $0,181 \mathrm{a}$ & $0,027 \mathrm{~b}$ & $0,006 \mathrm{a}$ & $0,187 \mathrm{a}$ \\
\hline $\mathrm{PC}$ & $1,51 \mathrm{a}$ & $0,417 \mathrm{a}$ & $0,036 \mathrm{~b}$ & $0,180 \mathrm{a}$ & $0,200 \mathrm{a}$ & $0,117 \mathrm{a}$ & $0,002 \mathrm{a}$ & $0,203 \mathrm{a}$ \\
\hline \multicolumn{9}{|c|}{ Profundidade - $75 \mathrm{~cm}$} \\
\hline PD & $1,46 \mathrm{~b}$ & $0,441 \mathrm{a}$ & $0,106 \mathrm{a}$ & $0,069 \mathrm{~b}$ & $0,266 \mathrm{a}$ & $0,039 \mathrm{~b}$ & $0,004 a$ & $0,271 \mathrm{a}$ \\
\hline PC & $1,56 \mathrm{a}$ & $0,392 b$ & $0,034 b$ & $0,170 \mathrm{a}$ & $0,188 \mathrm{~b}$ & $0,111 \mathrm{a}$ & $0,002 \mathrm{a}$ & $0,182 b$ \\
\hline
\end{tabular}

Valores seguidos da mesma letra em cada profundidade não diferem estatisticamente pelo teste de Tukey a $5 \%$ de probabilidade. Classe 1 - tamanho de mesoporos entre 50 e $8,9 \mu \mathrm{m}$; Classe 4 - tamanho de mesoporos entre 1,0 e 0,6 $\mu \mathrm{m}$

Tabela 2. Umidade na Capacidade de Campo (CC) e distribuição do tamanho de mesoporos em três classes para diferentes profundidades do solo sob plantio direto (PD) e preparo convencional $(P C)$

\begin{tabular}{|c|c|c|c|c|}
\hline \multirow{2}{*}{\multicolumn{2}{|c|}{ Tratamentos $\mathrm{CC}\left(\mathrm{cm}^{3} \mathrm{~cm}^{-3}\right)$}} & \multicolumn{3}{|c|}{ Distribuição do tamanho de mesoporos $\left(\mathrm{cm}^{3} \mathrm{~cm}^{-3}\right)$} \\
\hline & & Classe2 & Classe3 & Classe5 \\
\hline \multicolumn{5}{|c|}{ Sistemas de manejo do solo } \\
\hline PD & $0,264 b$ & $1,83 \times 10^{-2} a$ & $1,61 \times 10^{-2} \mathrm{~b}$ & $4,92 \times 10^{-3} \mathrm{a}$ \\
\hline PC & $0,284 \mathrm{a}$ & $2,53 \times 10^{-2} \mathrm{a}$ & $3,44 \times 10^{-2} a$ & $3,10 \times 10^{-3} \mathrm{~b}$ \\
\hline \multicolumn{5}{|c|}{ Profundidades no perfil $(\mathrm{cm})$} \\
\hline 2,5 & $0,278 \mathrm{~b}$ & $3,44 \times 10^{-2} a$ & $2,60 \times 10^{-2} \mathrm{a}$ & $4,33 \times 10^{-3} \mathrm{a}$ \\
\hline 7,5 & $0,278 b$ & $2,55 \times 10^{-2} a$ & $2,52 \times 10^{-2} \mathrm{a}$ & $3,93 \times 10^{-3} \mathrm{a}$ \\
\hline 15 & $0,260 \mathrm{bc}$ & $2,40 \times 10^{-2} \mathrm{a}$ & $1,56 \times 10^{-2} \mathrm{a}$ & $5,70 \times 10^{-3} \mathrm{a}$ \\
\hline 30 & $0,280 \mathrm{~b}$ & $2,28 \times 10^{-2} a$ & $3,36 \times 10^{-2} \mathrm{a}$ & $5,47 \times 10^{-3} \mathrm{a}$ \\
\hline 45 & $0,241 c$ & $1,87 \times 10^{-2} \mathrm{a}$ & $1,94 \times 10^{-2} \mathrm{a}$ & $3,66 \times 10^{-3} \mathrm{~b}$ \\
\hline 60 & $0,260 \mathrm{bc}$ & $1,44 \times 10^{-2} \mathrm{a}$ & $3,37 \times 10^{-2} a$ & $2,16 \times 10^{-3} \mathrm{~b}$ \\
\hline 75 & $0,318 \mathrm{a}$ & $1,27 \times 10^{-2} \mathrm{a}$ & $2,43 \times 10^{-2} a$ & $2,81 \times 10^{-3} \mathrm{~b}$ \\
\hline
\end{tabular}

Valores seguidos da mesma letra em cada profundidade não diferem estatisticamente pelo teste de Tukey a $5 \%$ de probabilidade. Classe 2 $=$ tamanho de mesoporos entre 8,9 e $2,9 \mu \mathrm{m}$; Classe $3=$ tamanho de mesoporos entre 2,9 e $1,0 \mu \mathrm{m}$; Classe $5=$ tamanho de mesoporos entre 0,6 e $0,2 \mu \mathrm{m}$

profundidades mais afetadas pelos sistemas de manejo (até $15 \mathrm{~cm}$ ) a diferença foi significativa somente naquela mais próxima à superfície, com porosidade total cerca de $17 \%$ mais alta em PD; este resultado também se mostra divergente em relação à tendência geral encontrada em grande parte dos trabalhos, nos mais diferentes tipos de solo (Stone \& Silveira, 2001; Tormena et al., 2004; Bescansa et al., 2006; Moret \& Arrúe,
2007). Para a macroporosidade e microporosidade, só nas maiores profundidades se observaram diferenças significativas entre sistemas de manejo, o que pode ser atribuído à variabilidade natural do solo, uma vez que o efeito de sistema se concentra nas camadas de solo próximas à superfície (Tormena et al., 2002).

Para a mesoporosidade, que é o tamanho de poros responsável pela retenção da água disponível às plantas, observouse ser mais elevada em $\mathrm{PD}$, próximo à superfície $(2,5 \mathrm{~cm})$, com diferença significativa em relação ao PC, apresentando variação direta com a densidade do solo (Tabela 1); esta tendência pode ter ocorrido devido ao maior teor de matéria orgânica nas camadas mais superficiais em PD, a qual proporciona maior agregação ao solo, mantendo uma estrutura mais estável e agregados mais resistentes possibilitando, inclusive, a redução da densidade do solo (Razafimbelo et al., 2008). Para as profundidades de 60 e $75 \mathrm{~cm}$, a diferença entre sistemas pode ser atribuída à variabilidade natural do solo. Nas demais profundidades não se constataram diferenças significativas entre PD e PC, para a mesoporosidade.

Considerando a importância da mesoporosidade para a retenção de água disponível às plantas, avaliou-se a distribuição dos mesoporos em cinco classes de tamanho. A interação foi significativa apenas nas classes 1 e 4 (Tabela 1), enquanto nas outras três não houve interação (Tabela 2). De maneira geral, em poucas situações a diferença foi significativa entre PD e PC (Tabelas 1 e 2) ou entre profundidades (Tabela 2). Próximo à superfície, na profundidade de $2,5 \mathrm{~cm}$, a diferença foi significativa na classe 1 (mesoporos entre 50 e $8,9 \mu \mathrm{m})$, com PD apresentando cerca do dobro de mesoporos 
que PC. Para as classes que não apresentaram interação, a diferença entre as profundidades não foi significativa, exceto para a classe 5, a partir da profundidade de $45 \mathrm{~cm}$, caso em que houve diferenças significativas apenas entre sistemas de manejo de solo, com a quantidade de mesoporos ora maior em PD, ora em PC (Tabela 2).

Ao se considerar somente a distribuição de mesoporos até $15 \mathrm{~cm}$ de profundidade, que é a camada de solo mais afetada pelo manejo, verificou-se que, em termos médios, a distribuição de mesoporos é diferente entre os sistemas de manejo (Figura 1), estando de acordo com resultados apresentados por Bescansa et al. (2006), os quais identificaram grandes alterações na distribuição de poros entre sistemas de manejo de solo, envolvendo o PD. Conforme a Figura 1, o PC apresentou uma distribuição de mesoporos que tendeu a uma curva normal, enquanto em PD a distribuição se aproximou de uma curva exponencial decrescente em relação ao tamanho de mesoporos, demonstrando que o solo em PD teve maior quantidade de mesoporos de maior diâmetro, nas classes próximas à macroporosidade mas no PC ocorreu maior quantidade de mesoporos com tamanhos intermediários, cujo resultado discorda daqueles de Bescansa et al. (2006), que encontraram maior quantidade de poros pequenos $(0,2-6 \mu \mathrm{m})$ em PD, em relação ao PC, porém concordam com aqueles apresentados por Oorts et al. (2007), em que o PC teve maior quantidade de pequenos poros em relação ao $\mathrm{PD}$, na camada de 0 a $5 \mathrm{~cm}$ de profundidade.

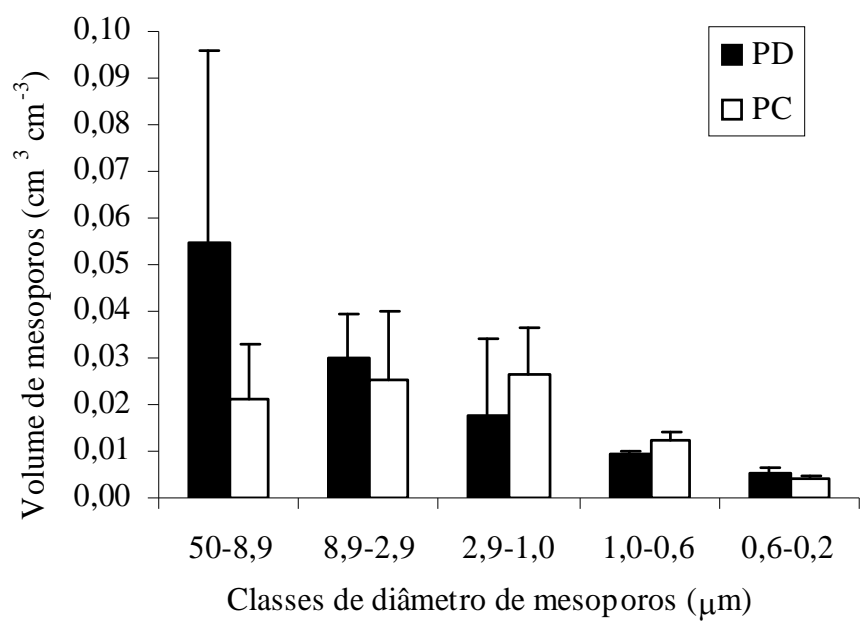

Figura 1. Distribuição da mesoporosidade média do solo entre 0 e $15 \mathrm{~cm}$ de profundidade, em plantio direto (PD) e preparo convencional (PC), segundo diferentes classes de diâmetro de mesoporos. As linhas sobre as barras correspondem ao desvio padrão

A diferença na distribuição do tamanho de mesoporos pode estar associada ao manejo que é dado ao solo, visto que o revolvimento frequente pulveriza o solo em PC, rompendo a conectividade e a estrutura dos mesoporos, aumentando o número de poros com tamanhos menores (Sauer et al., 1990; Malone et al., 2003), o que favorece a sua distribuição, segundo uma curva normal. Em PD, a maior quantidade de matéria orgânica (Bescansa et al., 2006) e a maior atividade de microrganismos e raízes, nas camadas mais próximas à superfície, com revolvimento mínimo do solo na semeadura, permi- tem um processo de auto-organização do sistema o qual, naturalmente, não é linear, o que explicaria a tendência observada na distribuição dos mesoporos.

Com relação à capacidade de campo $(\mathrm{CC})$ e o ponto de murcha permanente (PMP), que são os limites de água disponível às plantas, houve interação significativa somente para PMP. Na maioria das profundidades avaliadas foi notória a tendência de umidade do solo mais elevada no PMP em PC que em PD, mas com diferença significativa entre sistemas de manejo apenas na profundidade mais elevada (Tabela 1) porém não deve haver efeito de sistema de preparo do solo na profundidade de $75 \mathrm{~cm}$, sendo a diferença, portanto, atribuída à variabilidade natural do solo. Para a capacidade de campo (Tabela 2), a diferença foi significativa entre sistemas de manejo de solo e entre profundidades no perfil. A umidade na CC foi significativamente maior (cerca de 7\%) em PC em relação ao $\mathrm{PD}$, indicando que o solo em $\mathrm{PD}$ reteve menos umidade logo após a drenagem natural. Entre profundidades a umidade do solo na CC foi mais alta a $75 \mathrm{~cm}$ e teve menor valor a $45 \mathrm{~cm}$, enquanto próximo à superfície não houve diferenças significativas entre profundidades (Tabela 2). Esta tendência pode ser associada à presença de uma camada de transição para um horizonte B textural (Bt), a cerca de $45 \mathrm{~cm}$ de profundidade e ao próprio horizonte $\mathrm{Bt}$ a $75 \mathrm{~cm}$ de profundidade, com maior teor de argila que o restante do perfil (Rojas \& Lier, 1999). Reynolds et al. (2002) não encontraram diferenças de umidade do solo na CC e PMP entre PD e PC em vários tipos de solo, exceto para a $\mathrm{CC}$ em um dos solos avaliados. Segundo Reynolds et al. (2002), a falta de diferença significativa entre sistemas de manejo para a umidade do solo em CC e PMP, se deve ao fato de que a umidade do solo em PMP é determinada pelo conteúdo de argila, o qual não é afetado pelos sistemas de manejo enquanto aquela na CC resulta da interação complexa entre conteúdo de argila, estrutura, densidade e carbono orgânico do solo, cujo impacto da mudança desses fatores sobre a umidade do solo em CC, pode ser parcialmente compensado.

A análise de variância para retenção de água no solo (Figura 2) detectou interação significativa entre profundidades, potenciais aplicados e sistemas de manejo do solo. De modo geral, as diferenças significativas de retenção de água entre o solo em PD e PC foram mais frequentes em potenciais matriciais mais elevados $(-10 \mathrm{e}-33 \mathrm{kPa})$ do que em potenciais mais negativos, exceto para a profundidade de $75 \mathrm{~cm}$ (Figura 2). Na maioria das profundidades, a umidade do solo foi maior em PC que em PD, com a máxima diferença entre sistemas a $60 \mathrm{~cm}$, a qual foi de $29 \%$ no potencial de $-10 \mathrm{kPa}$. O solo em PD apresentou umidade mais elevada que em PC nos potenciais mais elevados e somente nas profundidades extremas (2,5 e $75 \mathrm{~cm}$ ) (Figura 2), que foram aquelas que apresentaram maior mesoporosidade (Tabela 1). Para potenciais de saturação e $-10 \mathrm{kPa}$, o solo em PD apresentou 13 e $17 \%$, respectivamente, mais umidade retida que em PC, a $2,5 \mathrm{~cm}$ de profundidade, sendo a diferença significativa apenas em -10 kPa. Essa condição também foi verificada por Rojas (1998), no terceiro ano de implantação dos sistemas PD e PC, na mesma área e provavelmente esteja relacionada à distribuição da mesoporosidade do solo (Figura 1), conforme discutido por Dalmago 
A.

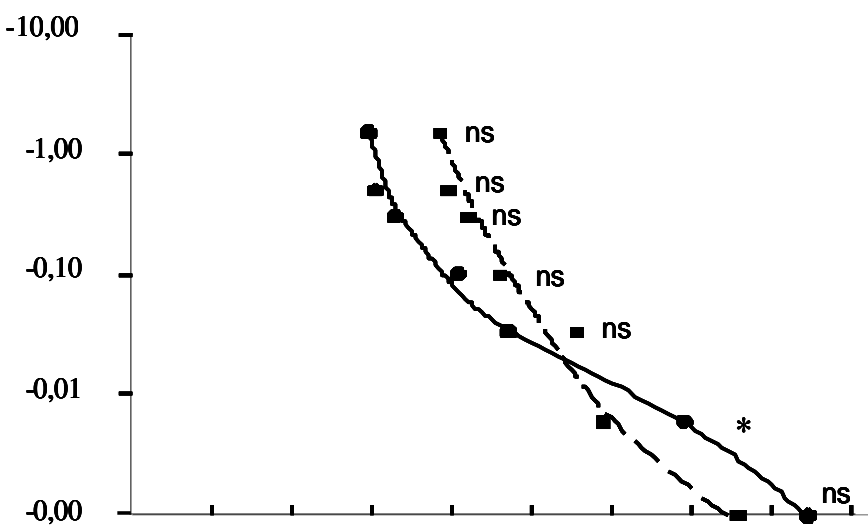

C.

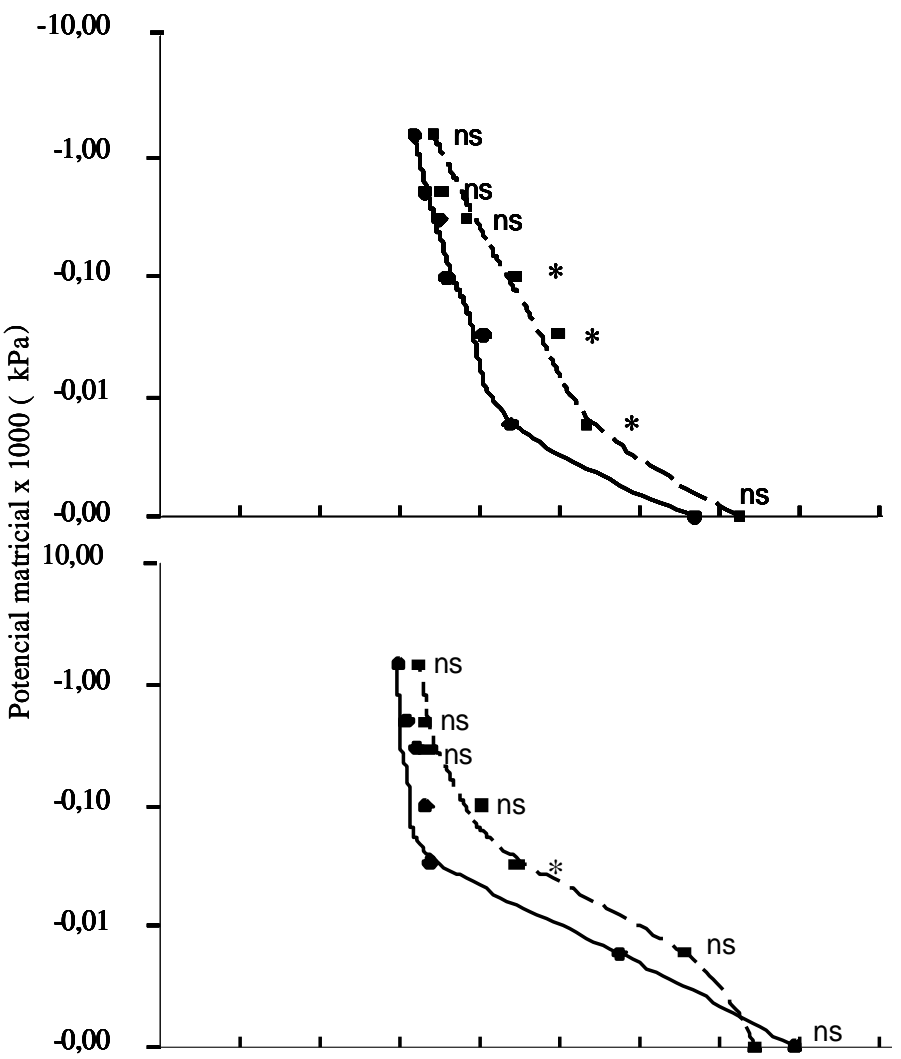

G.

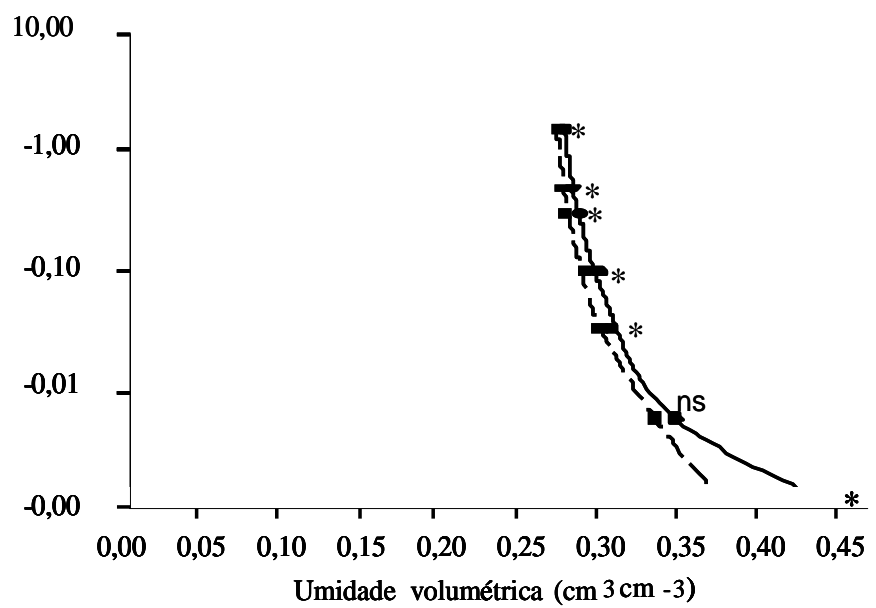

B.

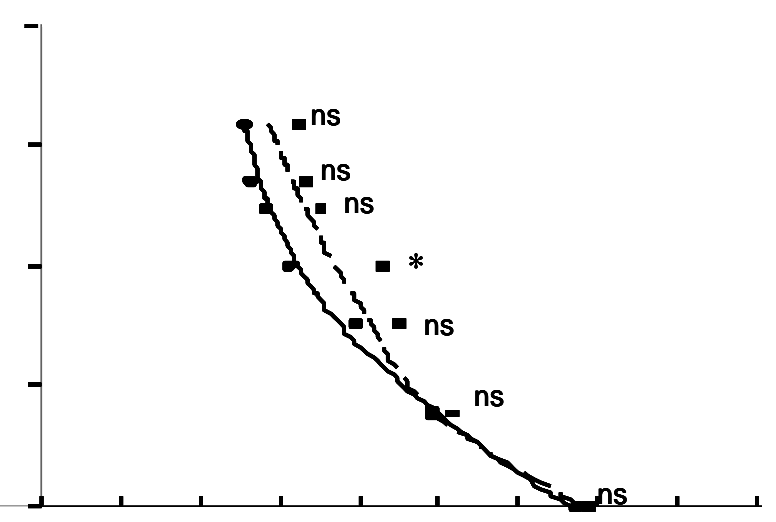

D.

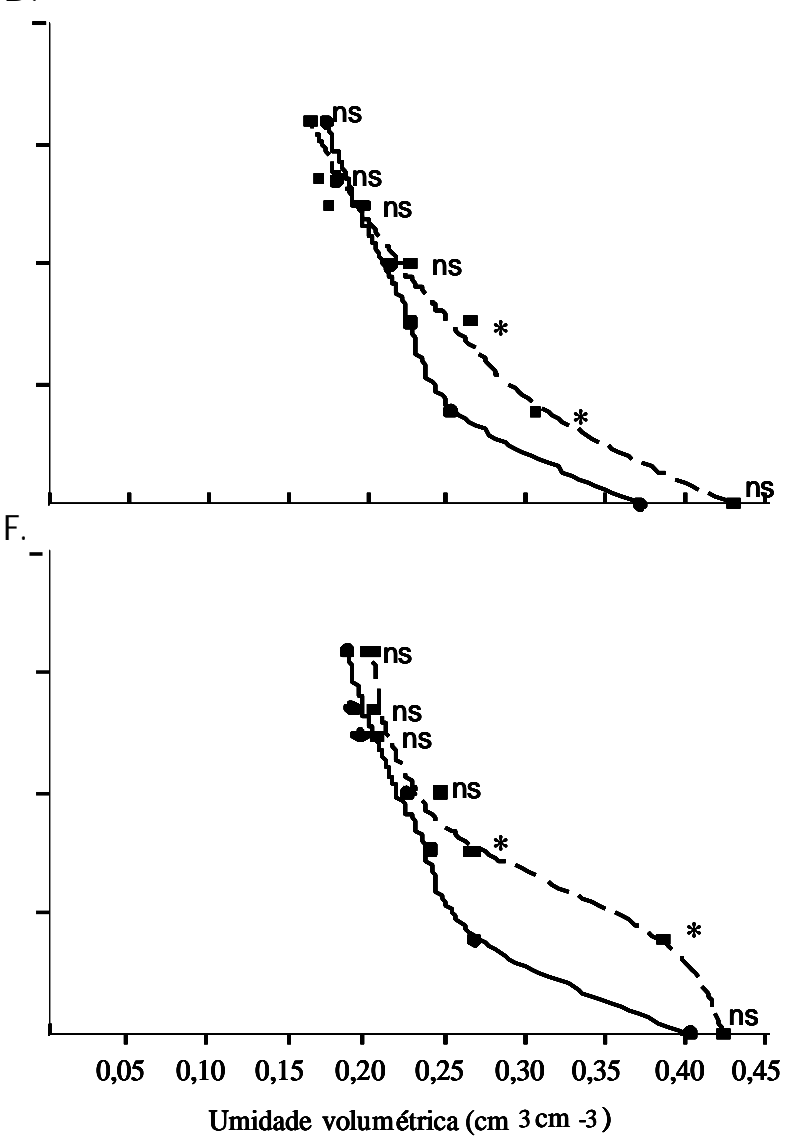

PD (Van Genuchten)

PC (Van Genuchten )

- PD (medido)

- $\quad$ PC (medido)

Figura 2. Curvas características de retenção de água no solo em plantio direto (PD) e preparo convencional (PC), nas profundidades de 2,5 (A), 7,5 (B), 15 (C), 30 (D), 45 (E), 60 (F) e $75 \mathrm{~cm}(\mathrm{G}$ ) e valores ajustados pelo M odelo de Van Genuchten (ns= diferenças não significativas em sistemas de manejo de solo pelo teste de Tukey a $5 \%$ de probabilidade, enquanto * significa diferenças significativas) 
A.

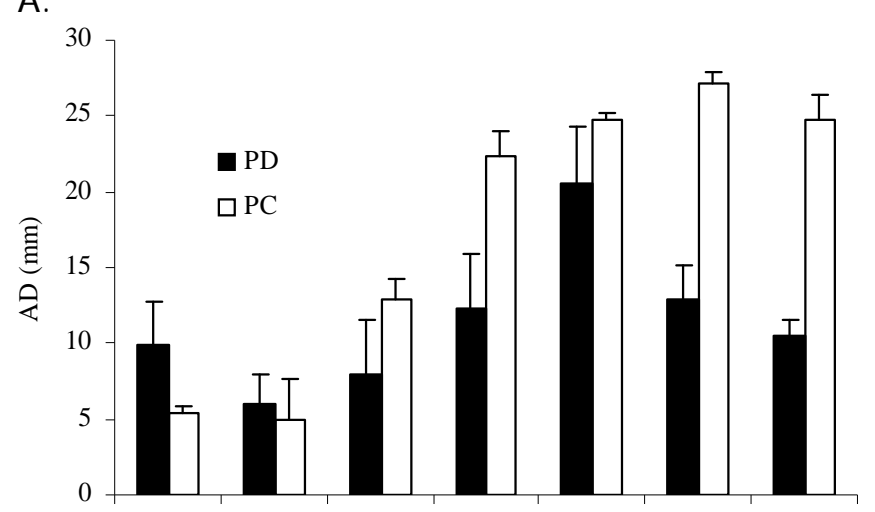

B.

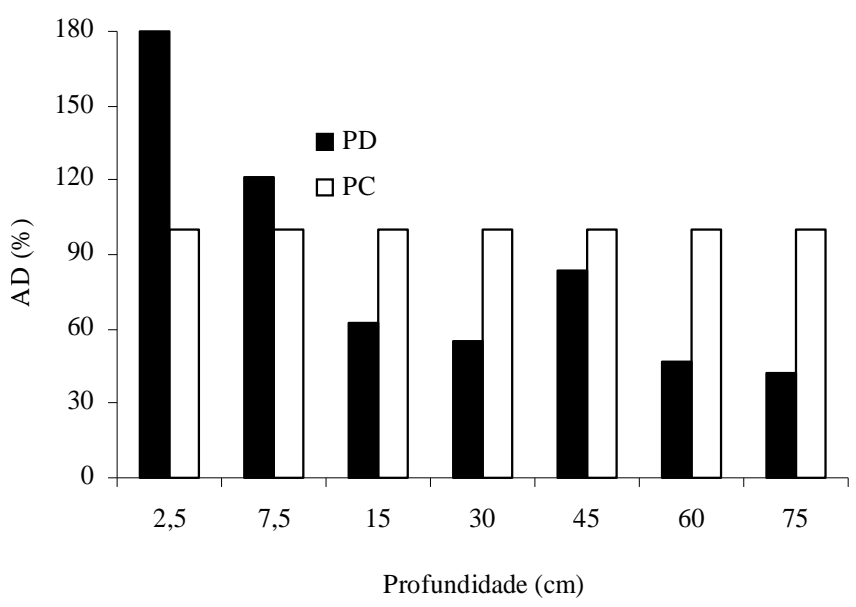

Figura 3. Água disponível (AD), em valor absoluto (A) e relativo (B), entre a capacidade de campo e o ponto de murcha permanente, para diferentes profundidades de solo em plantio direto (PD) e preparo convencional (PC)

(2004). Os parâmetros de ajuste das curvas para as diferentes profundidades e sistema de manejo de solo se encontram na Tabela 3.

Embora os resultados encontrados sejam coerentes, devese considerá-los com cautela, devido ao método de amostragem de solo. Amostras pontuais não permitem identificar a situação real de campo, principalmente quanto à geometria porosa do solo. A continuidade e a interligação entre os poros não são identificadas em razão do rompimento dos mesmos na coleta das amostras de solo; com isto, zonas com maior umidade, formadas pela incorporação de matéria orgâ- nica das raízes e atividade da fauna do solo, dificilmente são detectadas nos pequenos volumes de solo coletados, pelo qual a situação "in loco" pode diferir daquela projetada pelas análises de laboratório, sobretudo sob PD, pois as características de porosidade são diferentes e sua distribuição é mais complexa que aquela do solo em PC (Hubert et al., 2007) o que justificaria a umidade mais favorável ao desenvolvimento das plantas em PD, observada no campo (Salton \& Mielniczuk, 1995; Fabrizzi et al., 2005; Vita et al., 2007; Zanette et al., 2007).

A complexidade das relações entre porosidade de retenção de água no solo e sua utilidade aos vegetais, pode ser mais bem entendida pela análise da água disponível às plantas. Utilizando-se os limites de CC e PMP, verificou-se que a disponibilidade de água segue a mesma tendência da sua retenção (Figuras 3A e 3B), ou seja, maior em PC que em PD. Em termos absolutos, para a profundidade de $2,5 \mathrm{~cm}$ o solo em PD apresentou capacidade de $10 \mathrm{~mm}$ de água disponível às plantas $( \pm 1,97 \mathrm{~mm}$ por centímetro de profundidade), enquanto aquele em PC atingiu apenas $5,5 \mathrm{~mm}( \pm 1,1 \mathrm{~mm} \mathrm{~mm}$ por centímetro de profundidade de solo), ou seja, uma diferença acima de $80 \%$; já na profundidade de $7,5 \mathrm{~cm}$, esta diferença baixou para cerca de $20 \%$, com valores de $6 \mathrm{~mm}( \pm 1,21 \mathrm{~mm}$ por centímetro de profundidade de solo) e $5 \mathrm{~mm}( \pm 1,00 \mathrm{~mm}$ por $\mathrm{cm}$ de profundidade de solo) no solo em PD e PC, respectivamente (Figuras 3A e 3B).

A análise conjunta da disponibilidade de água às plantas entre os sistemas PD e PC, feita a partir da superfície do solo em direção ao extremo do perfil avaliado, mostra que nas duas primeiras profundidades o solo em PD apresentou aproximadamente $60 \%$ mais água disponível que em PC; já a $30 \mathrm{~cm}$ de profundidade, o solo em PC teve cerca de $84 \%$ mais água disponível que em PD mas, considerando-se a camada entre 0 e $30 \mathrm{~cm}$ de profundidade, o solo em PC apresentou mais água disponível às plantas $( \pm 45 \mathrm{~mm})$ que em PD ( $\pm 35 \mathrm{~mm})$ (Figura 4$)$, em aproximadamente $28 \%$, tendência esta correspondente à distribuição da matéria orgânica no perfil do solo obtida por Bona (2005) na mesma área pela qual, sob PD, a mesma se concentrou na superfície e nas camadas mais próximas à superfície. Sob PC e pelo efeito da aração, a matéria orgânica foi incorporada ao solo em profundidades em torno de $15 \mathrm{~cm}$.

Tabela 3. Coeficientes de ajuste da equação de Van Genuchten para solo sob plantio direto (PD) e preparo convencional (PC), em diferentes profundidades no perfil

\begin{tabular}{|c|c|c|c|c|c|c|c|c|c|c|}
\hline \multirow{3}{*}{$\begin{array}{l}\text { Profundidade } \\
\text { (cm) }\end{array}$} & \multicolumn{10}{|c|}{ Parâmetros de ajuste de equação de Van Genuchten } \\
\hline & \multicolumn{2}{|c|}{$\alpha\left(\mathrm{kPa}^{-1}\right)$} & \multicolumn{2}{|c|}{$\mathbf{m}$} & \multicolumn{2}{|c|}{$\mathbf{N}$} & \multicolumn{2}{|c|}{$\theta_{r}\left(\mathrm{~cm}^{3} \mathrm{~cm}^{-3}\right)$} & \multicolumn{2}{|c|}{$\theta_{\mathrm{s}}\left(\mathrm{cm}^{3} \mathrm{~cm}^{-3}\right)$} \\
\hline & PD & PC & PD & PC & PD & PC & PD & PC & PD & PC \\
\hline 2,5 & 909 & 465 & 0,436 & 0,347 & 1,772 & 1,531 & 0,147 & 0,193 & 0,422 & 0,379 \\
\hline 7,5 & 535 & 344 & 0,398 & 0,330 & 1,661 & 1,491 & 0,127 & 0,162 & 0,337 & 0,345 \\
\hline 15 & 114 & 292 & 0,318 & 0,310 & 1,466 & 1,450 & 0,159 & 0,171 & 0,334 & 0,362 \\
\hline 30 & 130 & 413 & 0,299 & 0,351 & 1,427 & 1,540 & 0,174 & 0,164 & 0,372 & 0,431 \\
\hline 45 & 840 & 1190 & 0,623 & 0,505 & 2,654 & 2,019 & 0,149 & 0,162 & 0,397 & 0,373 \\
\hline 60 & 167 & 1370 & 0,335 & 0,529 & 1,503 & 2,125 & 0,187 & 0,203 & 0,403 & 0,424 \\
\hline 75 & 227 & 1370 & 0,381 & 0,527 & 1,616 & 2,114 & 0,271 & 0,184 & 0,449 & 0,385 \\
\hline
\end{tabular}

OBS: $\mathrm{n}$ dependente de $\mathrm{m}$; $\theta \mathrm{r}$ e $\theta$ s fixos, obtidos por medições diretas 


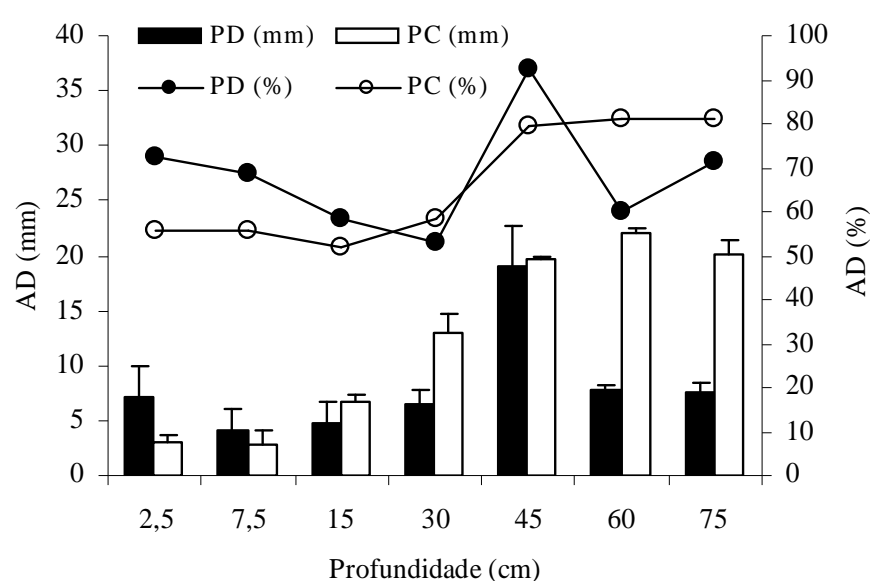

Figura 4. Água disponível (AD) no solo até o potencial matricial de $-80 \mathrm{kPa}$ $(\mathrm{mm})$ e valor relativo da mesma à capacidade máxima (\%), em diferentes profundidades de solo sob plantio direto (PD) e preparo convencional (PC)

A resposta apresentada neste trabalho indica uma provável correlação entre a disponibilidade de água encontrada e a distribuição da matéria orgânica no solo, avaliada por Bona (2005) na mesma área experimental. Tais evidências são reforçadas pelos resultados encontrados por Carpenedo \& Mielniczuk (1990), em que a maior armazenagem e disponibilidade de água às plantas em PD, nas camadas mais próximas à superfície do solo, estariam associadas a melhorias da estruturação do solo das primeiras camadas do perfil, sob PD, pela adição de matéria orgânica na superfície. A matéria orgânica influencia a mesoporosidade e a ampliação e/ou redução dos limites de CC e PMP (Reynolds et al., 2002) (Tabelas 1 e 2).

Embora o solo em PD tenha apresentado mais água disponível nas duas profundidades próximas à superfície e aquele em PC nas demais profundidades, a energia de retenção não seguiu a mesma tendência (Figura 4). Nas três primeiras profundidades do solo em PD, cerca de $70 \%$ do total de água disponível às plantas se encontravam armazenados até $-80 \mathrm{kPa}$ (limite de funcionamento dos tensiômetros), enquanto em PC somente $50 \%$ da água disponível estavam retidos até o mesmo limite. Considerando-se o total de água disponível até $45 \mathrm{~cm}$ de profundidade (Figura 4), camada que contém a maior parte das raízes das culturas anuais, conclui-se que o solo em PD ainda apresentou maior quantidade de água disponível às plantas até o limite de $-80 \mathrm{kPa}$ que em $\mathrm{PC}$, em torno de 73 e $64 \%$, respectivamente. Na medida em que se considerou o total de água disponível no perfil avaliado (até $75 \mathrm{~cm}$ de profundidade) as diferenças de energia de retenção desaparecem e ambos os sistemas de manejo apresentam em torno de $70 \%$ do total da água disponível até o limite de -80 kPa (Figura 4).

A diferença na quantidade de água disponível às plantas entre os sistemas de manejo do solo até o limite energético de $-80 \mathrm{kPa}$, é explicada pela distribuição do tamanho de mesoporos (Figura 1). A distribuição exponencial dos mesoporos em PD concentrando maior quantidade dos mesmos na classe de maior diâmetro $(50-8,9 \mu \mathrm{m})$, proporciona que mais água seja retida com menor energia em comparação com a distribuição normal dos mesoporos no solo em PC, cuja maior quantidade de mesoporos esteve na classe de diâmetro médio $(2,9-1,0 \mu \mathrm{m})$ (Figura 1). Considerando que as forças de retenção de água no solo são mais fortes na superfície dos poros que na parte intermediária, poros de maior tamanho têm menor força de atração deixando a água mais livre e disponível às plantas; isto significa que as plantas cultivadas em PD absorvem água do solo com maior facilidade em relação àquelas em PC, principalmente em condição de alta demanda evaporativa da atmosfera (Bianchi, 2004) e quando as raízes ainda se concentram nas camadas superficiais do solo. A maior facilidade em absorver água proporciona um estado hídrico mais favorável às plantas em PD, retardando o início do déficit hídrico em uma condição de estiagem, conforme observado por (Bianchi et al., 2005; Bianchi et al., 2007), na mesma área experimental e trabalhando com milho.

\section{CONCLUSÕES}

1. A retenção de água no solo em plantio direto é maior que no solo em preparo convencional nas camadas mais próximas à superfície e em potenciais matriciais mais elevados.

2. A disponibilidade de água às plantas no solo em plantio direto é maior que no solo em preparo convencional nas camadas mais próximas à superfície, seguindo a tendência observada para a retenção de água no solo.

3. A distribuição exponencial decrescente dos mesoporos no solo em plantio direto com maior frequência em diâmetros maiores determina menor energia de retenção da água disponível às plantas que no solo em preparo convencional, cuja mesoporosidade segue uma curva de distribuição normal.

\section{AGRADECIMENTOS}

Os autores agradecem ao $\mathrm{CNPq}$, pelo apoio financeiro na execução do trabalho de campo e pelas bolsas de doutorado de Genei A. Dalmago, produtividade em pesquisa de Homero Bergamaschi, iniciação científica de Flávia Comiran e Bruna M. M. Heckler, e à Capes, pela Bolsa de Mestrado de Cleusa A. M. Bianchi. Agradecem, também, a FEPAGRO (Fundação Estadual de Pesquisa Agropecuária) pela cedência de equipamentos e laboratórios.

\section{LITERATURA CITADA}

Baumhardt, R. L.; Jones, O. R. Residue management and tillage effects on soil-water storage and grain yield of dryland wheat and sorghum for a clay loam in Texas. Soil \& Tillage Research, v.68, p.71-82, 2002.

Bergamaschi, H.; Guadagnin, M. R.; Cardoso, L. S.; Silva, M. I. G. da. Clima da estação experimental da UFRGS (e região de abrangência). Porto Alegre: UFRGS, 2003. 78p. 
Bescansa, P.; Imaz, M. J.; Virto, I.; Enrique, A.; Hoogmoed, W. B. Soil water retention as affected by tillage and residue management in semiarid Spain. Soil \& Tillage Research, v.87, p.19-27, 2006.

Bianchi, C. A. M. Avaliação de indicadores da condição hídrica em milho sob diferentes níveis de água, em semeadura direta e convencional. Porto Alegre: UFRGS, 2004. 98p. Dissertação Mestrado

Bianchi, C. A. M.; Bergonci, J. I.; Bergamaschi, H.; Dalmago, G. A. Ajuste osmótico em milho cultivado em diferentes sistemas de manejo de solo e disponibilidade hídrica. Pesquisa Agropecuária Brasileira, v.40, n.7, p.645-651, 2005.

Bianchi, C. A. M.; Bergonci, J. I.; Bergamaschi, H.; Dalmago, G. A.; Heckler, B. M. M.; Comiran, F. Condutância da folha em milho cultivado em plantio direto e convencional em diferentes disponibilidades hídricas. Ciência Rural, v.37, n.2, p.315332, 2007.

Blake, G. R.; Hartge, K. H. Bulk Density. In: Klute, A. (ed.) Methods of soil analysis: Part 1 - Physical and mineralogical methods. Madison: SSSA, 1986. p.363-375. Agronomy, Monographic 9

Bona, F. D. de. Dinâmica da matéria orgânica do solo em sistemas irrigados por aspersão sob plantio direto e preparo convencional. Porto Alegre: UFRGS, 2005. 150p. Dissertação Mestrado

Carpenedo, V.; Mielniczuk, J. Estado de agregação e qualidade de agregados de Latossolos Roxos, submetidos a diferentes sistemas de manejo. Revista Brasileira de Ciência do Solo, v.14, p.99-105, 1990.

Cruz, A. C. R.; Pauletto, E. A.; Flores, C. A.; Silva, J. B. Atributos físicos e carbono orgânico de um argissolo vermelho sob sistemas de manejo. Revista Brasileira de Ciência do Solo, v.27, p.1105-1112, 2003.

Dalmago, G. A. Dinâmica da água no solo em cultivos de milho sob plantio direto e preparo convencional. Porto Alegre: UFRGS, 2004. 245p. Tese Doutorado

Drury, C. F.; Tan, C.; Welacky, T. W.; Oloya, T. O.; Hamill, A. S.; Weaver, S. E. Red clover and tillage influence on soil temperature, water content, and corn emergence. Agronomy Journal, v.91, p.101-108, 1999.

Fabrizzi, K. P.; García, F. O.; Costa, J. L.; Picone, L. I. Soil water dynamics, physical properties and corn and wheat responses to minimum and no-tillage systems in the southern Pampas of Argentina. Soil \& Tillage Research, v.81, p.57-69, 2005.

Hanks, R. J.; Sisson, D. V.; Hurst, R. L.; Hubbard, K. G. Statistical analysis of results from irrigation experiments using the line-source sprinkler system. Soil Science Society of America Journal, v.44, p.886-888, 1980.

Hatfield, J. L.; Sauer, T. J.; Prueger, J. H. Managing soils to achieve greater water use efficiency: a review. Agronomy Journal, v.93, p.271-280, 2001.

Hillel, D. Enviromental soil physics. San Diego: Academic Press, 1998, 771p.

Hubert, F.; Hallaire, V.; Sardini, P.; Caner, L.; Heddadj, D. Pore morphology changes under tillage and no-tillage practices. Geoderma, v.142, p.226-236, 2007.
Kay, B. D.; Vandenbygaart, A. J. Conservation tillage and depth stratification of porosity and soil organic matter. Soil \& Tillage Research, v.66, p.107-118, 2002.

Lampurlanés, J.; Angás, P.; Cantero-Martínez, C. Root growth, soil content and yield of barley under different tillage systems on two soils in semiarid conditions. Field Crops Research, v.69, p.27-40, 2001.

Libardi, P. L. Dinâmica da água no solo. Piracicaba: P. L. Libardi, 1995. 497p.

Malone, R. W.; Longsdon, S.; Shipitalo, M. J.; Weatherington-Rice, J.; Ahuja, L.; Ma, L. Tillage effect on macroporosity and herbicide transport in percolate. Geoderma, v.116, p.191-215, 2003.

Masri, Z.; Ryan, J. Soil organic matter and related physical properties in a Mediterranean wheat-based rotation trial. Soil \& Tillage Research, v.87, p.146-154, 2006.

Model, N. S.; Levien, R.; Frosi, R. A. Água armazenada e temperatura do solo em oito sistemas de manejo do solo. Pesquisa Agropecuária Gaúcha, v.1, p.41-49, 1995.

Moret, D.; Arrúe, J. L. Dynamics of soil hydraulic properties during fallow as affected by tillage. Soil \& Tillage Research, v.96, p.103-113, 2007.

Oorts, K.; Bossuyt, H.; Labreuche, J.; Merckx, R.; Nicolardot, B. Carbono and nitrogen stocks in relation to organic matter fractions, aggregation and pore size distribution in no-tillage and conventional tillage in northern France. European Journal of Soil Science, v.58, p.248-259, 2007

Osunbitan, J. A.; Oyedele, D. J.; Adekalu, K. O. Tillage effects on bulk density, hydraulic conductivity and strength of a loamy sand soil in southwestern Nigéria. Soil \& Tillage Research, v.82, p.57-64, 2005.

Rasiah, V.; Aylmore, L. A. G. Caracterizing the changes in soil porosity by computer tomography and fractal dimension. Soil Science, v.163, p.203-211, 1998.

Razafimbelo, T. M.; Albrecht, A.; Oliver, R.; Chevallier, T.; Chapuis-Lardy, L.; Feller, C. Aggregate associated-C and physical protection in a tropical clayey soil under Malagasy conventional and no-tillage systems. Soil \& Tillage Research, v.98, p.140-149, 2008.

Reynolds, W. D.; Bowman, B. T.; Drury, C. F.; Tan, C. S.; Lu, X. Indicators of good soil physical quality: density and storage parameters. Geoderma, v.110, p.131-146, 2002.

Rojas, C. A. L. Alterações físico-hídricas de um podzólico em função do manejo de solo. Porto Alegre: UFRGS, 1998. 77p. Dissertação Mestrado

Rojas, C. A. L.; Lier, Q. J. van. Alterações físicas e hídricas de um podzólico em função de sistemas de preparo. Pesquisa Agropecuária Gaúcha, v.5, p.105-115, 1999.

Salton, J. C.; Mielniczuk, J. Relações entre sistemas de preparo, temperatura e umidade de um Podzólico Vermelho-escuro de Eldorado do Sul (RS). Revista Brasileira de Ciência do Solo, v.19, p.313-2319, 1995.

Sauer, T. J.; Clothier, B. E.; Daniel, T. C. Surface measurements of the hydraulic properties of a tilled and untilled soil. Soil \& Tillage Research, v.15, p.359-369, 1990.

Secco, D.; Ros, C. O. da; Secco, J. K.; Fiorin, J. E.; Atributos físicos e produtividade de culturas em um Latossolo Vermelho Agiloso sob diferentes sistemas de manejo. Revista Brasileira de Ciência do Solo, v.29, p.407-414, 2005. 
Shukla, M. K.; Lal, R.; Ebinger, M. Tillage effects on physical and hydrological properties of a typic argioquoll in Central Ohio. Soil Science, v.168, n.11, p.802-811, 2003.

Stone L. F.; Silveira, P. M. Efeitos do sistema de preparo e da rotação de culturas na porosidade e densidade do solo. Revista Brasileira de Ciência do Solo, v.25, p.395-401, 2001.

Tollner, E. W.; Hargrove, W. L.; Langdale, G. W. Influence of conventional and no-tillage practices on soil physical properties in the southern Piedmont. Journal of Soil and Water Conservation, v.39, p.73-76, 1984.

Tormena, C. A.; Barbosa, M. C.; Costa, A. C. S. da; Gonçalves, A. C. A. Densidade, porosidade e resistência à penetração em Latossolo cultivado sob diferentes sistemas de preparo do solo. Scientia Agrícola, v.59, n.4, p.795-801, 2002.

Tormena, C. A.; Roloff, G.; Sá, J. C. M. Propriedades físicas do solo sob plantio direto influenciado por calagem, preparo inicial e tráfego. Revista Brasileira de Ciência do Solo, v.22, p.301-309, 1998.
Tormena, C. A.; Silva, A. P. Incorporação da densidade no ajuste de dois modelos à curva de retenção de água no solo. Revista Brasileira de Ciência do Solo, v.26, p.305-314, 2002.

Tormena, C. A.; Vidigal Filho, P. S.; Gonçalves, A. C. A.; Araújo, M. A.; Pinto, J. C. Influência de diferentes sistemas de preparo do solo nas propriedades físicas de um Latossolo Vermelho distroférico. Revista Brasileira de Engenharia Agrícola e Ambiental, v.8, n.1, p.65-71, 2004.

Vita, P. de; Paolo, E. D.; Fecondo, G.; Fonzo, N. D.; Pisante, M. No-tillage and convencional tillage effects on durun wheat yield, grain quality and soil moisture content in southern Italy. Soil \& Tillage Research, v.92, p.69-78, 2007.

$\mathrm{Xu}, \mathrm{D}$.; Mermound, A. Modelling the soil water balance based on timedependent hydraulic conductivity under different tillage practices. Agricultural Water Management, v.63, p.139-151, 2003.

Zanette, S. V.; Sampaio, S. C.; Silvestre, M. G.; Boas, M. A. V.; Uribe-Opazo, M. A.; Queiroz, M. M. F. de. Análise espacial da umidade do solo cultivado com soja sob dois sistemas de manejo Revista Brasileira de Engenharia Agrícola e Ambiental, v.11, n.3, p.239-247, 2007. 